\title{
Optimizion of enzymatic hydrolysis of zein to prepare protein hydrolysate with increased antioxidants.
}

\author{
Hai-Teng Tao ${ }^{1,2,3}$, Yu-Xiao Wang ${ }^{1,2,3}$, Wei Liu ${ }^{1,2,3}$, Bin Qiu ${ }^{1,2,3 *}$, Li-Na Liu ${ }^{1,2,3}$, Ai-Zhen Zong ${ }^{1,2,3}$, Min \\ Jia ${ }^{1,2,3}$, Tong-Cheng $\mathrm{Xu}^{1,2,3 *}$ \\ ${ }^{1}$ Institute of Agro-Food Science and Technology, Shandong Academy of Agricultural Sciences, PR China \\ ${ }^{2}$ Key Laboratory of Agro-Products Processing Technology of Shandong Province, Ministry of Agriculture, 202 Gongye \\ North Road, Jinan 250100, PR China \\ ${ }^{3}$ Key Laboratory of Novel Food Resources Processing, Ministry of Agriculture, 202 Gongye North Road, Jinan 250100, \\ PR China
}

\begin{abstract}
Background: Protein hydrolysate was prepared with zein recycled from corn gluten meal. It have been reported that zein have the antioxidant activity. Various recycling methods were selected to obtain zein with desirable characteristics, but ignored the antioxidant activity of hydrolysate. No comparisons of different recycling methods of zein or studies on the use of RSM to optimize enzymatic hydrolysis conditions to increase antioxidant activity could be found.

Materials and methods: The corn gluten meal used in this study was a gift from the Xiwang group in China. Zein was dis-colored from corn gluten meal by alcohol (1:6) under ultrasound (Elmasonic S50R, German) twice for 15 min each time. Zein was extracted through preliminary optimized conditions of $55 \%$ ethanol at $38^{\circ} \mathrm{C}$ for $15 \mathrm{~min}$. The extracted zein was placed in a beaker before use.

Results: The optimum conditions to obtain a high DPPH radical scavenging rate was close to the predicted value $(7.523 \%, 0.0416 \mathrm{mg} / \mathrm{mL}$ ascorbate equiv), and they were as follows: an enzyme to substrate level of $1 \%(\mathrm{w} / \mathrm{w})$, temperature of $40^{\circ} \mathrm{C}$, time of one hour, and $\mathrm{pH}$ of 8 . HPLC detected more than 26 constituents in the zein hydrolysate, and the content of glutamic acid rose at a relatively high degree from 16.8 to 19.1 .

Conclusion: The best recycling method for colourless zein with high antioxidant activity was dilutionfreeze-drying. The zein including hydrolysate has potential for applications in functional foods, which would significantly improve the added value.
\end{abstract}

Keywords: Recycling, Zein, DPPH radical scavenging rate, Enzymatic hydrolysis.

Accepted on May 21, 2018

\section{Introduction}

The film-forming properties of zein, the principal protein of corn named by John Gorham in 1821, make it popular for coating preservation or sub-micron structured encapsulation $[1,2]$. At present, the main method of preparing zein is aqueous alcohol extraction from discolored corn gluten meal (CGM). The production of zein has been well-researched; however, the current prohibitive costs of bleaching and extraction have limited its application. Recent studies have also reported antioxidant activity as a result of the hydrolysis of zein. Through a powder model system of docosahexaenoic acid ethyl ester (DHE), Matsumura et al. found that zein rich in $\beta$ and $\gamma$-type subunits had higher antioxidant activities [3]. Both glutamine mild deamidation and glutamic acid/glutamate residual formations may cause zein to have radical scavenging activity. Concurrently, reports have confirmed that the acid or alkali treatment of the conversion of glutamine and asparagine amino acids to their acidic or salt forms could enhance antioxidant activity [4]. Zhang et al. researched the antioxidant properties of zein at different $\mathrm{pH}$ levels, reporting that the radical scavenging rate at $\mathrm{pH} 2.7$ and 12.5 was higher than that at $\mathrm{pH} 3.3,6.5$ and 10.5 [5]. Although zein had antioxidant activity in aqueous alcohol, the high content of non-polar amino acids, such as alanine, proline, and leucine, made it difficult to dissolve zein in water. However, enzymatic hydrolysis could increase the solubility of zein in both aqueous alcohol and water. Enzyme treatment may enhance the functional properties, such as solubility, foaming, and moisture absorption, of zein in general [4].

Recent research has demonstrated specific enzymatic treatments that could effectively enhance the antioxidant activity of zein and its hydrolysate. For example, Zheng et al. 
found that the enzymatic hydrolysis of extruded corn gluten possessed better solubility and antioxidant activity than without enzymatic treatment [6]. Different enzymatic hydrolysis processes could cause varied levels of antioxidant activity, as evidenced by Kong et al. discovering that alcalasehydrolyzed zein had a stronger antioxidant activity than papain-hydrolyzed zein [7].

The literature available relates antioxidant activity to relieve many chronic diseases. Antioxidant activity could have a direct effect on the reduction of radicals or could indirectly consume substances that easily generate free radicals. DPPH antioxidant assay has been used frequently in testing the stability of antioxidants. Previous studies primarily focused on the degree of hydrolysis or the yield rate, but ignored the antioxidant activity of hydrolysate [8]. No comparisons of different recycling methods of zein or studies on the use of RSM to optimize enzymatic hydrolysis conditions to increase antioxidant activity could be found. Therefore, the present work focused on optimizing recycling methods and enzymatic hydrolysis conditions in order to stimulate antioxidant activity.

\section{Materials and Methods}

\section{Materials}

The corn gluten meal used in this study was a gift from the Xiwang group in China. DPPH was purchased from Sigma. Alcalase (endoproteinase from Bacillus licheniformis, 2.4 AU/g) was bought from Novozymes. Unless mentioned otherwise, the chemicals and reagents used in this experiment were analytical grade.

\section{Methodology}

Zein was discolored from CGM by alcohol (1:6) under ultrasound (Elmasonic S50R, German) twice for $15 \mathrm{~min}$ each time. Zein was extracted through preliminary optimized conditions of $55 \%$ ethanol at $38^{\circ} \mathrm{C}$ for $15 \mathrm{~min}$. The extracted zein was placed in a beaker before use.

\section{Recycling methods}

Freeze-drying (FD): The zein extract was freeze-dried at $-80^{\circ} \mathrm{C}$ for $24 \mathrm{~h}$ (FD-1B-80, Beijing Boyikang experimental medical instrument Co., Ltd.).

Vacuum-drying (VD): The zein extract was vacuum-dried at $45^{\circ} \mathrm{C}$ for $24 \mathrm{~h}$ below $0.085 \mathrm{MPa}$ (D2F-6050, Shanghai Jing Hong laboratory instrument Co., Ltd.).

Blast-drying (BD): Zein extract was desiccated at $100^{\circ} \mathrm{C}$ for 24 h (DHG9240, Shanghai Jing Hong laboratory instrument Co., Ltd.).

Dilution-freeze-drying (DFD): Cold water $\left(0-4^{\circ} \mathrm{C}\right)$ was added to the zein extract to dilute the alcohol concentration to $20-40 \%$. At this concentration, the zein extract was in a state between coacervation and precipitation [8]. After removing the residual pigment, cold water was added to produce precipitate. The following treatments were the same as FD.
Refrigeration-vacuum-drying (RVD): The zein extract was flocculated at $-18^{\circ} \mathrm{C}$. After removing supernatant, the following treatments were the same as VD.

\section{The yield, purity, color and DPPH radical scavenging rate}

The yield and the purity of the zein were measured, based on previous studies, with a Kjeldahl auto analyzer (Hanon K9860, American) $[9,10]$. A calculation of the percentage of extracted zein weight from the total CGM weight $(\mathrm{db})$ was used to determine the yield rate of recycled zein. The protein purity was calculated as the amount of protein content in the extracted zein.

The Lovibond Tintometer, a color comparison instrument (WSL-2, Shanghai precision scientific instrument Co., Ltd.), was used to determine the color of the zein. The versatile and visual colorimeter measures the color of liquids, solids, powders and pastes with Lovibond red, yellow, blue and neutral units [11].

The DPPH assay was created based on the literature from previous research [12], but with some modifications. $0.1 \mathrm{~mL}$ of zein solution $(1 \mathrm{mg} / \mathrm{mL})$ and $3.9 \mathrm{~mL}$ of DPPH $(1 \mathrm{mM})$ in a $55 \%$ ethanol solution were mixed and then placed in the dark for $30 \mathrm{~min}$ at room temperature. The absorbance was examined with a spectrophotometer (UV-1750, SHIMADZU) set at 517 $\mathrm{nm}$. A standard curve (linear) was generated for comparison by reacting $0.1 \mathrm{~mL}$ of a concentration made of different ascorbates $(0-0.2 \mathrm{mg} / \mathrm{mL})$ with $3.9 \mathrm{~mL}$ of DPPH solution.

\section{The preparation of zein hydrolysate}

The zein hydrolysate was prepared by using a vortex mixer (lab dancer, Germany IKA) to throughly blend the alcalase and then reacting the solution in a water bath. After hydrolysis, the solution was heated at $95^{\circ} \mathrm{C}$ for $4 \mathrm{~min}$ to inactivate the enzyme. The zein hydolysate solution was centrifuged (CR22GIII HighSpeed Refrigerated Centrifuge, Hitachi Koki Co., Ltd) at 4000 $\mathrm{r} / \mathrm{min}$ for $4 \mathrm{~min}$ and after, the supernatant was freeze-dried and stored at $0-4^{\circ} \mathrm{C}$ before use.

\section{Optimization experiments}

The conditions for the optimization experiments were in alignment with previous methods $[12,13]$. SAS software was used to generate the factorial design, (consisting of four factors, three levels, and three center points, as shown in Table 1a), for the optimization experiments. Table $1 \mathrm{~b}$ shows the results of the optimization experiments (Table 1c).

Table 1a. Factors and levels of response surface design.

\begin{tabular}{lllll}
\hline \multirow{2}{*}{ Factor } & Code & \multicolumn{2}{c}{ Levels } \\
\cline { 3 - 5 } & & -1 & 0 & 1 \\
\hline $\mathrm{E} / \mathrm{S}, \mathrm{w} / \mathrm{w}$ & $\mathrm{X}_{1}$ & 0.01 & 0.02 & 0.03 \\
\hline Temperature,${ }^{\circ} \mathrm{C}$ & $\mathrm{X}_{2}$ & 40 & 50 & 60 \\
\hline
\end{tabular}




\begin{tabular}{lllll}
\hline Time, hour & $\mathrm{X}_{3}$ & 1.5 & 2 & 2.5 \\
\hline $\mathrm{pH}$ & $\mathrm{X}_{4}$ & 8 & 8.5 & 9 \\
\hline
\end{tabular}

Table 1b. Design and results of response surface experiments.

\begin{tabular}{|c|c|c|c|c|c|}
\hline Run & $\mathbf{x}_{1}$ & $\mathbf{x}_{2}$ & $\mathbf{x}_{3}$ & $\mathbf{X}_{4}$ & $Y$ \\
\hline 1 & 0.01 & 40 & 2 & 8.5 & 2.343 \\
\hline 2 & 0.01 & 60 & 2 & 8.5 & 4.311 \\
\hline 3 & 0.03 & 40 & 2 & 8.5 & 4.592 \\
\hline 4 & 0.03 & 60 & 2 & 8.5 & 5.061 \\
\hline 5 & 0.02 & 50 & 1.5 & 8 & 6.373 \\
\hline 6 & 0.02 & 50 & 1.5 & 9 & 1.687 \\
\hline 7 & 0.02 & 50 & 2.5 & 8 & 5.623 \\
\hline 8 & 0.02 & 50 & 2.5 & 9 & 2.812 \\
\hline 9 & 0.01 & 50 & 2 & 8 & 6.279 \\
\hline 10 & 0.01 & 50 & 2 & 9 & 2.53 \\
\hline 11 & 0.03 & 50 & 2 & 8 & 5.436 \\
\hline 12 & 0.03 & 50 & 2 & 9 & 5.623 \\
\hline 13 & 0.02 & 40 & 1.5 & 8.5 & 4.967 \\
\hline 14 & 0.02 & 40 & 2.5 & 8.5 & 4.217 \\
\hline 15 & 0.02 & 60 & 1.5 & 8.5 & 5.061 \\
\hline 16 & 0.02 & 60 & 2.5 & 8.5 & 5.155 \\
\hline 17 & 0.01 & 50 & 1.5 & 8.5 & 4.873 \\
\hline 18 & 0.01 & 50 & 2.5 & 8.5 & 5.248 \\
\hline 19 & 0.03 & 50 & 1.5 & 8.5 & 5.248 \\
\hline 20 & 0.03 & 50 & 2.5 & 8.5 & 5.248 \\
\hline 21 & 0.02 & 40 & 2 & 8 & 6.935 \\
\hline 22 & 0.02 & 40 & 2 & 9 & 3.187 \\
\hline 23 & 0.02 & 60 & 2 & 8 & 5.623 \\
\hline 24 & 0.02 & 60 & 2 & 9 & 3.468 \\
\hline 25 & 0.02 & 50 & 2 & 8.5 & 4.03 \\
\hline 26 & 0.02 & 50 & 2 & 8.5 & 4.03 \\
\hline 27 & 0.02 & 50 & 2 & 8.5 & 3.655 \\
\hline
\end{tabular}

$\mathrm{X}_{1}$ : Enzyme to substrate level; $\mathrm{X}_{2}$ : Temperature; $\mathrm{X}_{3}$ : Time; $\mathrm{X}_{4}$ : $\mathrm{pH} ; \mathrm{Y}$ : DPPH radical scavenging rate, $\%$.

Table 1c. ANOVA for radical scavenging rate of master model.

\begin{tabular}{llllll}
\hline Source & DF & SS & MS & F & Pr>F \\
\hline $\mathrm{X}_{1}$ & 1 & 2.635781 & 2.635781 & 3.730826 & 0.0774 \\
\hline $\mathrm{X}_{2}$ & 1 & 0.49532 & 0.49532 & 0.701103 & 0.4188 \\
\hline $\mathrm{X}_{3}$ & 1 & 0.000736 & 0.000736 & 0.001042 & 0.9748 \\
\hline $\mathrm{X}_{4}$ & 1 & 23.97579 & 23.97579 & 33.93661 & $<0.0001$
\end{tabular}

\begin{tabular}{llllll}
\hline $\mathrm{X}_{1} \times \mathrm{X}_{1}$ & 1 & 1.249365 & 1.249365 & 1.768419 & 0.2083 \\
\hline $\mathrm{X}_{1} \times \mathrm{X}_{2}$ & 1 & 0.56175 & 0.56175 & 0.795131 & 0.3901 \\
\hline $\mathrm{X}_{1} \times \mathrm{X}_{3}$ & 1 & 0.035156 & 0.035156 & 0.049762 & 0.8272 \\
\hline $\mathrm{X}_{1} \times \mathrm{X}_{4}$ & 1 & 3.873024 & 3.873024 & 5.482086 & 0.0373 \\
\hline $\mathrm{X}_{2} \times \mathrm{X}_{2}$ & 1 & 0.333333 & 0.333333 & 0.471818 & 0.5052 \\
\hline $\mathrm{X}_{2} \times \mathrm{X}_{3}$ & 1 & 0.178084 & 0.178084 & 0.25207 & 0.6247 \\
\hline $\mathrm{X}_{2} \times \mathrm{X}_{4}$ & 1 & 0.634412 & 0.634412 & 0.897981 & 0.362 \\
\hline $\mathrm{X}_{3} \times \mathrm{X}_{3}$ & 1 & 1.075205 & 1.075205 & 1.521903 & 0.2409 \\
\hline $\mathrm{X}_{3} \times \mathrm{X}_{4}$ & 1 & 0.878906 & 0.878906 & 1.244051 & 0.2865 \\
\hline $\mathrm{X}_{4} \times \mathrm{X}_{4}$ & 1 & 0.587861 & 0.587861 & 0.83209 & 0.3796 \\
\hline Model & 14 & 35.07731 & 2.505522 & 3.54645 & 0.0170 \\
\hline Error & 12 & 8.477848 & 0.706487 & & \\
\hline Lack of fit & 10 & 8.384098 & 0.83841 & 17.88608 & 0.0541 \\
\hline Pure error & 2 & 0.09375 & 0.046875 & & \\
\hline Total & 26 & 43.55515 & & & \\
\hline
\end{tabular}

\section{The purification, HPLC, and amino acid analysis of zein hydrolysate}

The zein hydrolysate was purified by column chromatography at a flow rate of $1 \mathrm{~mL} / \mathrm{min}$ with a Sephadex G-25 (GE healthcare bio-sciences AB, Sweden). The mobile phase consisted of ultrapure water, which was collected every $10 \mathrm{~min}$ in a small tube. Next, the antioxidant activity of the sample in each tube was tested by DPPH assay. Afterward, the collected sample with highest antioxidant activity was analyzed by high performance liquid chromatography (Aglient 1200 serious, USA) with a $300 \mathrm{SB}$ C18 column $(4.6 \times 150 \mathrm{~mm}$ i.d., $5 \mu \mathrm{m})$. The mobile phase during the elution consisted of water containing $0.1 \%$ trifluoroacetic acid (Eluent A) and of acetonitrile containing $0.1 \%$ trifluoroacetic acid (Eluent B). The optimized conditions with this column were as follows: flow rate of $0.5 \mathrm{~mL} / \mathrm{min}, 5-40 \%$ B for $35 \mathrm{~min}$, injected volume of $5 \mu \mathrm{L}, \lambda=280 \mathrm{~nm}$, and temperature of $30^{\circ} \mathrm{C}$. Amino acid analysis was completed according to the Chinese National Standard GB/T 5009.124-2003.

\section{Statistical analysis}

The Least Significant Difference (LSD) method was used to make multiple comparisons between the data results, with $\alpha=0.01$ performed in 3 independent replications. The results of the response surface method were analyzed with SAS 9.2.

\section{Results and Discussion}

\section{Comparisons of different recycling processes}

Table 2 shows that the purity level, treated as the total protein in the extracted zein, varied in different recycling processes. The purity level was lowest from the vacuum drying process, and was at its highest level from the blast-drying and dilution- 
freeze-drying processes. These results occurred because some of the non-zein matter decomposed in high temperature or washed away by water. The yield rate of recovered zein was highest from the blast drying process, but the standard deviation reached $3.41 \%$, primarily as a result of the temperature. High temperature had a negative effect on the zein, and increased the margin of error for the yield rate. Certain conditions, caused by differences in solvents and materials, caused the yield rate to be higher than the yield in experiments done by $\mathrm{Wu}$ et al. $[9,10]$. DPPH radical scavenging activity was highest under the condition of vacuum drying. The other recycling methods, with the exception of freeze-drying, had no significant impact on DPPH radical scavenging activity. The color of the zein in the fourth recycling method was obviously lighter than the zein produced from the other recycling methods. Instead of directly freezedrying, adding cold water to the extraction in the dilutionfreeze-drying method could cause the zein to coacervate and precipitate, leading to the easy removal of the residual pigment. This method using precipitate would conserve more energy than directly freeze-drying. The fourth method had the light color, and also guaranteed the yield, purity and antioxidant.

Table 2. The characteristics of zein as a result of different recycling methods.

\begin{tabular}{|c|c|c|c|c|c|c|c|}
\hline & \multirow{2}{*}{ Purity, \% } & \multirow{2}{*}{ Yield, \% } & \multicolumn{3}{|l|}{ Color } & \multicolumn{2}{|c|}{ Radical scavenging activity } \\
\hline & & & Yellow & Red & Blue & Rate, \% & Ascorbate equiv. $\mathrm{mg} / \mathrm{mL}$ \\
\hline FD & $88.27 \pm 0.69^{b}$ & $25.6 \pm 0.37^{a b}$ & 7 & 2 & 2 & $2.926 \pm 0.383^{b}$ & 0.0162 \\
\hline VD & $85.21 \pm 0.07^{c}$ & $27.4 \pm 0.14^{\mathrm{ab}}$ & 8 & 3.3 & 3.7 & $4.097 \pm 0.442^{\mathrm{a}}$ & 0.0226 \\
\hline $\mathrm{BD}$ & $90.04 \pm 0.94^{a}$ & $27.7 \pm 3.41^{\mathrm{a}}$ & 7 & 3.4 & 4 & $3.540 \pm 0.460^{\mathrm{ab}}$ & 0.0196 \\
\hline DFD & $90.81 \pm 0.39^{a}$ & $26.3 \pm 0.14^{\mathrm{ab}}$ & 2 & 0.8 & 0.8 & $3.651 \pm 0.210^{\mathrm{ab}}$ & 0.0202 \\
\hline RVD & $86.83 \pm 0.74^{b c}$ & $23.6 \pm 0.23^{b}$ & 7 & 2.8 & 3.5 & $3.456 \pm 0.294^{a b}$ & 0.0191 \\
\hline
\end{tabular}

abcMeans ( \pm standard deviations, $n=3$; each with duplicate measurements) without the same letter differ significantly ( $\alpha=0.01)$.

\section{Optimization of enzymatic hydrolysis parameters}

As Table 1c shows only $\mathrm{pH}$ level was extremely significant $(\mathrm{p}<0.01)$ among these independent variables. The interactions between the different factors did not have a significant influence $(p>0.05)$ on any of the response variables except for the interaction between the enzyme level and the $\mathrm{pH}$ level $(p<0.05)$. The model was logical, because the model was significant $(p<0.05)$ while the lack of fit was not significant $(p>0.05)$. The order of relevance of primary factors is $\mathrm{pH}>$ enzyme level $>$ temperature $>$ time. The model equation used for calculating the radical scavenging rate was as follows:

$\mathrm{Y}=236.1727-15.94658 \times \mathrm{X}_{1}-0.916158 \times \mathrm{X}_{2}-24.84083 \times$ $\mathrm{X}_{3}-37.0715 \times \mathrm{X}_{4}+0.484 \times \mathrm{X}_{1}^{2}+0.0025 \times \mathrm{X}_{2}^{2}+1.796 \times$ $\mathrm{X}_{3}^{2}+1.328 \times \mathrm{X}_{4}^{2}-0.037475 \times \mathrm{X}_{1} \times \mathrm{X}_{2}-0.1875 \times \mathrm{X}_{1} \times$ $\mathrm{X}_{3}+1.968 \times \mathrm{X}_{1} \times \mathrm{X}_{4}+0.0422 \times \mathrm{X}_{2} \times \mathrm{X}_{3}+0.07965 \times \mathrm{X}_{2} \times \mathrm{X}_{4}$ $+1.875 \times \mathrm{X}_{3} \times \mathrm{X}_{4}$.

According to Ridge Analysis, the Eigen values were namely $0.938,0.610,0.235$ and -0.268 , so the stationary point was a saddle point. Optimizing the hydrolysis condition according to Tang et al. did not result in a maximum value $[12,13]$. Nevertheless, the hydrolysis condition could still be optimized through the use of the master model $(\mathrm{p}<0.05)$. The yield of zein hydrolysate was 10 to $20 \%$, but the DPPH radical scavenging rate of the zein hydrolysate was obviously higher than that of the zein. Table $1 \mathrm{~b}$ shows that the $21^{\text {st }}$ experiment had the highest radical scavenging rate of $6.935 \%$, which was equal to $0.0383 \mathrm{mg} / \mathrm{mL}$ of ascorbate. Maintaining the $\mathrm{pH}$ at 8 optimized the radical scavenging rate, and for the most optimal results, the conditions were as follows: enzyme to substrate level $1 \%$ (w/w), temperature at $40^{\circ} \mathrm{C}$, time at one hour, and a $\mathrm{pH}$ of 8 . Under these conditions, the DPPH radical scavenging rate was $7.523 \%(0.0416 \mathrm{mg} / \mathrm{mL}$ ascorbate equiv. $)$, which was close to the predicted value.

\section{Analysis of zein hydrolysate}

The zein hydrolysate was purified by column chromatography through the use of a Sephadex G-25. As Figure 1 demonstrates, the fraction was typically washed out in 30 to $90 \mathrm{~min}$ (Table 3); the fraction that absorbed the most in $65-75$ min possessed a higher DPPH radical scavenging rate than the other fractions. Special amino acids (e.g. tryptophan, tyrosine and phenylalanine) are necessary for a high absorbancy rate at 280 $\mathrm{nm}$, so the highest antioxidant fractions contained at least one of these amino acids with the benzene ring structure. As a result, this fraction was analyzed by HPLC.

Figure 2 shows that there were more than 26 constituents present in the hydrolysate. The antioxidant activity of the zein hydrolysate was higher than that of the zein itself because the zein was hydrolyszed into groups of small fragments, some of which were exposed to the system. The constituents differed from Ma et al. in both types and content [14]. The next step was to completely separate all the constituents and then to collect each constituent according to varying polar coordinates. More details about each component still needed to be studied by other methods, such as quadrupole time of flight mass spectrometry or nuclear magnetic resonance. 


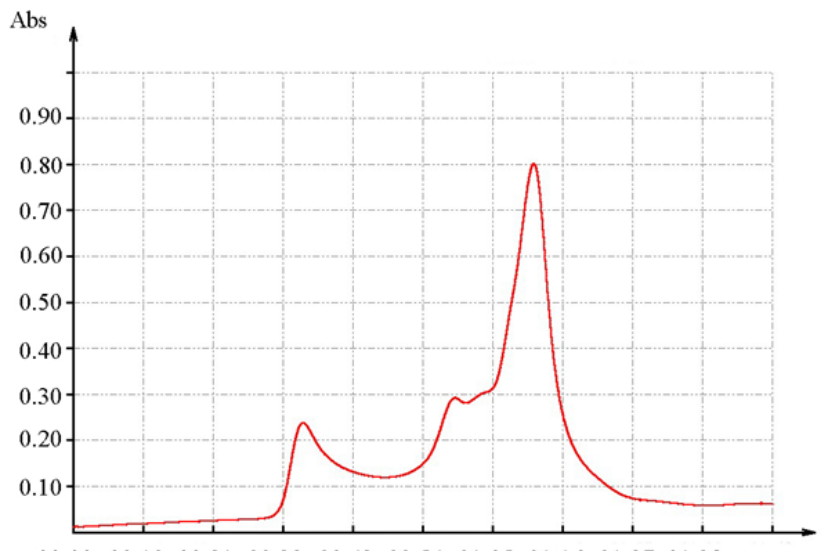

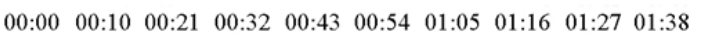

Figure 1. Column chromatography, with Sephadex $G-25$, at $1 \mathrm{~mL} / \mathrm{min}$ of ultrapure water. The fractions were collected in a tube every 10 min.

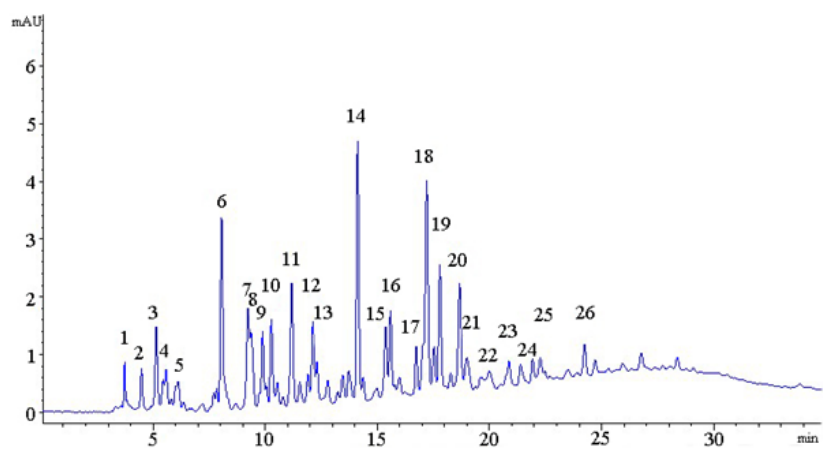

Figure 2. HPLC was performed using a mobile phase consisting of water $+0.1 \%$ trifluoroacete acid (Eluent A) and acetonitrile $+0.1 \%$ trifluotoacete acid (Eluent B). Gradient elution was carried out according to the following process: $0-35 \mathrm{~min}, \mathrm{~B}, 5-40 \%$.

As shown in Table 4, the content of total amino acids rose from 77.9 to 86.7 after hydrolysis, but the contents of the individual amino acids rose at varying degrees. Most significantly, glutamic rose the highest degree from 16.8 to 19.1 . This result was consistent with the conclusions on the antioxidant activity of zein hydrolasate from previous studies, which proposed that the increased radical scavenging rate may be attributed to the mild deamidation of glutamine and to the formation of residual glutamic acid/glutamatere in zein [5].

Table 3. DPPH radical scavenging rate in different course.

\begin{tabular}{lll}
\hline & Course, $\min$ & Radical scavenging rate, $\%$ \\
\hline 1 & 42125 & 0.03 \\
\hline 2 & $15-25$ & 0.05 \\
\hline 3 & $25-35$ & 0.76 \\
\hline 4 & $35-45$ & 1.15 \\
\hline 5 & $45-55$ & 2.01 \\
\hline 7 & $55-65$ & 3.03 \\
\hline
\end{tabular}

\begin{tabular}{lll}
\hline 8 & $75-85$ & 0.56 \\
\hline 9 & $85-95$ & 0.24
\end{tabular}

Note: At the beginning, it was mobile phase washed out from the column. The fractions were collected from the 5 th $\mathrm{min}$.

Table 4. Amino acid composition of zein and its hydrolysate.

\begin{tabular}{lll}
\hline Amino acid & Zein & Zein hydrolasate \\
\hline Aspargine/aspartate & 3.35 & 3.97 \\
\hline Glutamic & 16.8 & 19.1 \\
\hline Serine & 3.92 & 4.33 \\
\hline Glycine & 1.4 & 1.36 \\
\hline Histidine & 0.7 & 0.81 \\
\hline Arginine & 1.24 & 1.3 \\
\hline Threonine & 1.55 & 1.95 \\
\hline Alanine & 7.71 & 8.52 \\
\hline Proline/hydroxy proline & 8.56 & 8.3 \\
\hline Tyrosine & 3.78 & 4.3 \\
\hline Valine & 15.2 & 17.1 \\
\hline Methionine & 6.92 & 3.23 \\
\hline L-Cystine & 1.06 & 1.26 \\
\hline Isoleucine & 0.25 & 0.86 \\
\hline Leucine & 3.15 & 3.47 \\
\hline Phenyl alanine & 0.21 & 0.07 \\
\hline Lysine & 86.7 \\
\hline Total amino acid & & \\
\hline & & \\
\hline
\end{tabular}

\section{Conclusion}

The best recycling method for colorless zein with high antioxidant activity was dilution-freeze-drying. The optimal hydrolysis conditions to obtain increased antioxidant activity were: enzyme to substrate level $1 \%(\mathrm{w} / \mathrm{w})$, temperature of $40^{\circ} \mathrm{C}$, time of $1 \mathrm{~h}$, and a $\mathrm{pH}$ of 8 . Through HPLC analysis, more than 26 types of zein hydrolysate constituents were detected. After hydrolasate, total amino acids increased from 77.9 to 86.7 and, with the exception of glycine, proline and lysine, most amino acids increased. The zein including hydrolysate has potential for applications in functional foods, which would significantly improve the added value.

\section{Acknowledgement}

The authors gratefully acknowledge the grant from the National High Technology Research and Development Program of China (863 Program, No. 2013AA102206). 


\section{References}

1. Neo YP, Ray S, Jin J, Gizdavic-Nikolaidis M, Nieuwoudt M K, Liu D, Quek S Y, Encapsulation of food grade antioxidant in natural biopolymer by electro-spinning technique: a physicochemical study based on zein-gallic acid system, Food Chem 2013; 136: 1013-1021.

2. Gomez-Estaca J, Balaguer MP, Gavara R, HernandezMunoz P. Formation of zein nanoparticles by electrohydrodynamic atomization: Effect of the main processing variables and suitability for encapsulating the food coloring and active ingredient curcumin. Food Hydrocolloid 2012; 28: 82-91.

3. Matsumura Y, Andonova P P, Hayashi Y, Murakami H, Mori T. Antioxidant activities of zeins from different maize varieties against docosahexaenoic acid ethyl ester. Cereal Chem 1994; 71: 428-433.

4. Shukla R, Cheryan M. Zein: the industrial protein from corn. Ind Crop Prod 2001; 13: 171-192.

5. Zhang B, Luo Y, Wang Q. Effect of acid and base treatments on structural, rheological, and antioxidant properties of $\alpha$-zein. Food Chem 2011; 124: 210-220.

6. Zheng X, Li L, Liu X, Wang X, Lin J, Li D. Production of hydrolysate with antioxidative activity by enzymatic hydrolysis of extruded corn gluten. Appl Microbiol Biot 2006; 73: 763-770.

7. Kong B, Xiong YL, Antioxidant activity of zein hydrolysates in a liposome system and the possible mode of action. J Agr Food Chem 2006; 54: 6059-6068.

8. Wang Y, Padua GW. Formation of zein microphases in ethanol-water. Langmuir 2010; 26: 12897-12901.

9. Wu S, Myers DJ, Johnson LA. Factors affecting yield and composition of zein extracted from commercial corn gluten meal. Cereal Chem 1997; 74: 258-263.

10. Wu S, Myers DJ, Johnson LA. Effects of maize hybrid and meal drying conditions on yield and quality of extracted zein. Cereal Chem 1997; 74: 268-273.
11. Patil MM, Kalse SB, Jain SK. OSMO-convective drying of onion slices. Res J Recent Sci 2012; 1: 51-59.

12. Zhu L, Chen J, Tang X, Xiong LY. Reducing, radical scavenging, and chelation properties of in vitro digests of alcalase-treated zein hydrolysate. J Agr Food Chem 2008; 56: 2714-2721.

13. Tang X, He Z, Dai Y, Xiong LY, Xie M, Chen J. Peptide fractionation and free radical scavenging activity of zein hydrolysate. J Agr Food Chem 2009; 58: 587-593.

14. Ma ZL, Zhang WJ, Yu GC, He H, Zhang Y. The primary structure identification of a corn peptide facilitating alcohol metabolism by HPLC-MS/MS. Peptides 2012; 37: 138-143.

\section{*Correspondence to}

Bin Qiu

Institute of Agro-Food Science and Technology

Shandong Academy of Agricultural Sciences

Ministry of Agriculture

202 Gongye North Road

Jinan

PR China

Tong-Cheng Xu

Institute of Agro-Food Science and Technology

Shandong Academy of Agricultural Sciences

Ministry of Agriculture

202 Gongye North Road

Jinan

PR China 